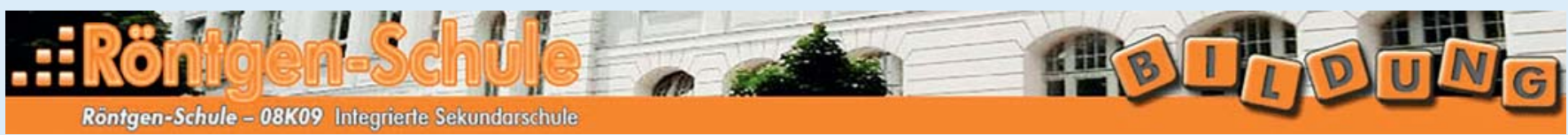

\section{Bildung mit Röntgen}

Im Jahr 2016 war die Integrierte-Röntgen-Sekundarschule aus Berlin-Neukölln an die Deutsche Röntgengesellschaft mit der Bitte herangetreten, ein Unterrichtsprojekt zu Wilhelm Conrad Röntgen zu unterstützen. Inzwischen sind aus diesem Erstkontakt vielfältige Formen der Zusammenarbeit erwachsen, die insbesondere denjenigen zugutekommen, um die es geht: Schülerinnen und Schüler der Röntgenschule.

Am Anfang stand ein Arbeitskreis. Lehrerinnen und Lehrer der Integrierten-RöntgenSekundarschule in Berlin-Neukölln sowie die Kunsthistorikerin Dr. Vera Dünkel hatten es sich zur Aufgabe gemacht, die Schülerinnen und Schüler zum Namensgeber der Schule zu informieren und Angebote zu schaffen, die auf spielerische und künstlerische Weise die Geschichte und das Prinzip der Röntgenstrahlen vermitteln sollten. Ziel war es, den aus sechs Berliner Stadtbezirken kommenden Schülern Identifikationsmuster anzubieten, die ihnen eine Inte-

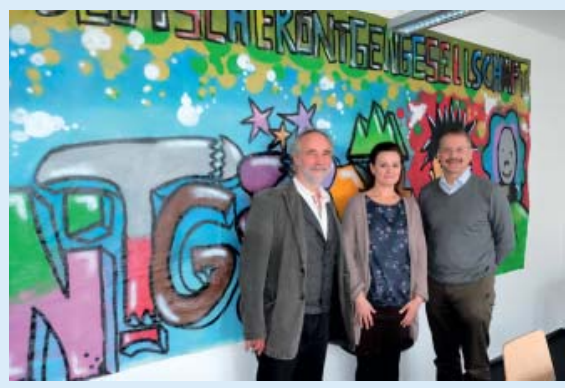

Paul A. Kleinert von der Röntgenschule in Berlin-Neukölln zu Gast in der DRG-Geschäftsstelle. Im Hintergrund ein Graffiti, das Schülerinnen und Schüler eigens für die DRG angefertigt haben.

gration vor Ort erleichtern und sie befähigen, sich als „Schüler der Röntgenschule“ zu fühlen.

Entstanden ist das Projekt „Bilder durch Röntgen“, das nach einer ersten Erprobungsphase ab dem Schuljahr 2016/2017 in den schulischen Regelbetrieb überführt und Anfang 2017 als Unterrichtsprojekt in die Tat umgesetzt wurde.

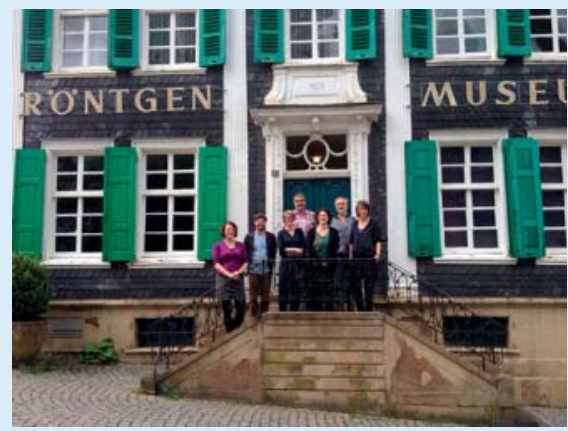

Die Mitglieder des Arbeitskreises Wilhelm Conrad Röntgen der Röntgenschule in Berlin-Neukölln zu Besuch im Deutschen Röntgen-Museum.

\section{Schule am anderen Ort}

Aus der einmaligen Unterstützung der DRG für das Projekt „Bilder durch Röntgen“ sind im weiteren Verlauf des Jahres 2017 ein regelmäßiger Austausch und intensivere Formen der Zusammenarbeit entstanden.

In den Mittelpunkt rückte dabei die Idee, Schülerfahrten nach Remscheid-Lennep zu 

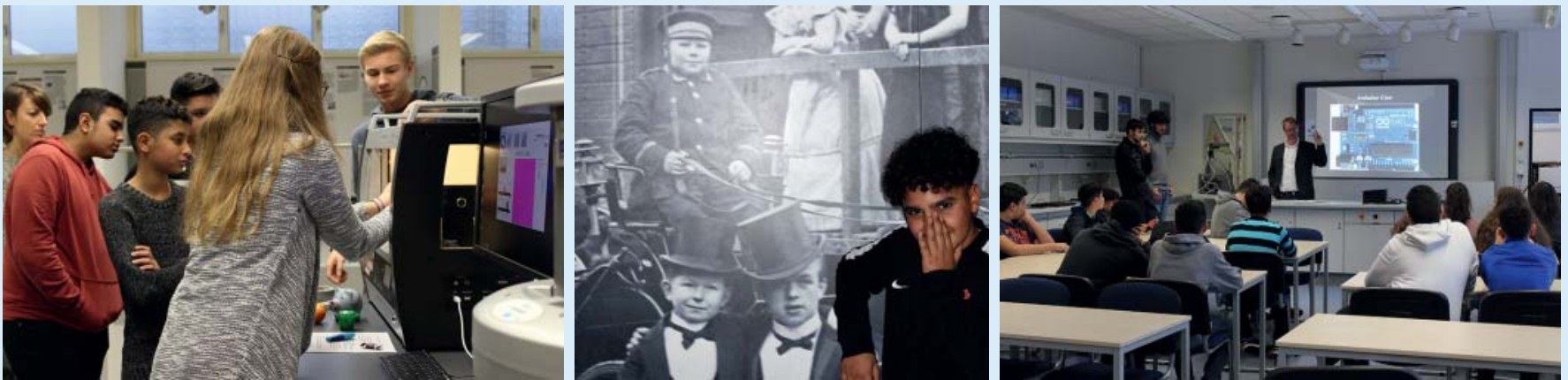

Neugierde und Forschergeist prägten den Aufenthalt der Berliner Schülerinnen und Schüler in Remscheid-Lennep.
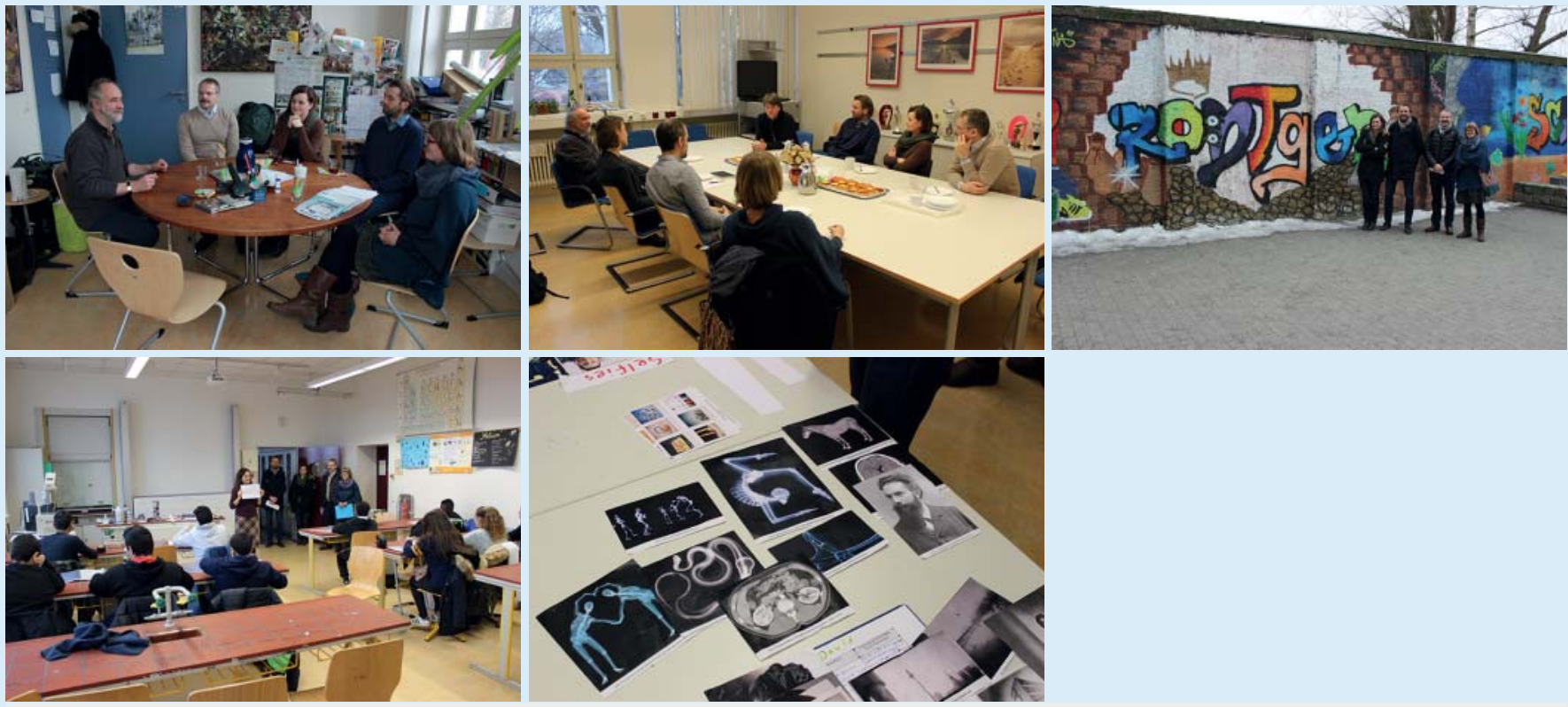

organisieren. Der Vorstand der DRG befürwortete im März 2017 eine entsprechende Förderung durch die Fachgesellschaft und im Juni machten sich die Mitglieder des Arbeitskreises Wilhelm Conrad Röntgen für erste Sondierungen auf den Weg nach Nordrhein-Westfalen. Eine künftige Zusammenarbeit wurde mit dem Deutschen Röntgen-Museum, dem Röntgen-Gymnasium in Lennep, der Stadtverwaltung Remscheid sowie mit dem Jugendclub „Welle“ vereinbart.

Vom 11. bis 14. Dezember 2017 fand die erste Exkursion unter dem Titel „Schule am anderen Ort“ mit Röntgenschülern aus Berlin statt. Das Programm war äußerst vielfältig und beinhaltete $u$. a. einen Bildungsparcours im Deutschen Röntgen-Museum sowie den Besuch des Röntgen-Schülerlabors (RöLab). Betreut wurden die Berliner
Schüler hier von Mentoren des Kurses „Medizinische Physik“ des Röntgen-Gymnasiums. Eine zweite Exkursion ist für das Frühjahr 2018 fest eingeplant.

\section{Sophie trifft Wilhelm Conrad}

Ebenfalls von der DRG gefördert wird ein Gemeinschaftsprojekt der IntegriertenRöntgen-Sekundarschule und der SophieBrahe-Gemeinschaftsschule in Berlin-Treptow. Sophie Brahe (1556-1643) war die Schwester des dänischen Astronomen Tycho Brahe und selbst eine der wichtigsten Gelehrten der frühen Neuzeit, die auf Gebieten der Literatur, Medizin, Botanik, Chemie, Mathematik und Astronomie bewandert war. Zusammen mit ihrem Bruder führte sie genaueste Himmelsbeobachtungen und astronomische Berechnungen durch, verfasste einen Fixsternkatalog und studierte 1572 die Erscheinung einer Supernova - ein entscheidender Schritt auf dem Weg vom geozentrischen zum heliozentrischen Weltbild.

Ziel des Projekts „Sophie trifft Wilhelm Conrad“ ist die Erarbeitung und Aufführung eines Theaterstücks, in dem beide Persönlichkeiten fiktiv miteinander in Dialog treten. Über die Verschränkung der Lebensläufe von Röntgen und Brahe werden Lebensprozesse aufgegriffen, die sich für Identifikationsabläufe der Schüler der beiden Schulen anbieten. 

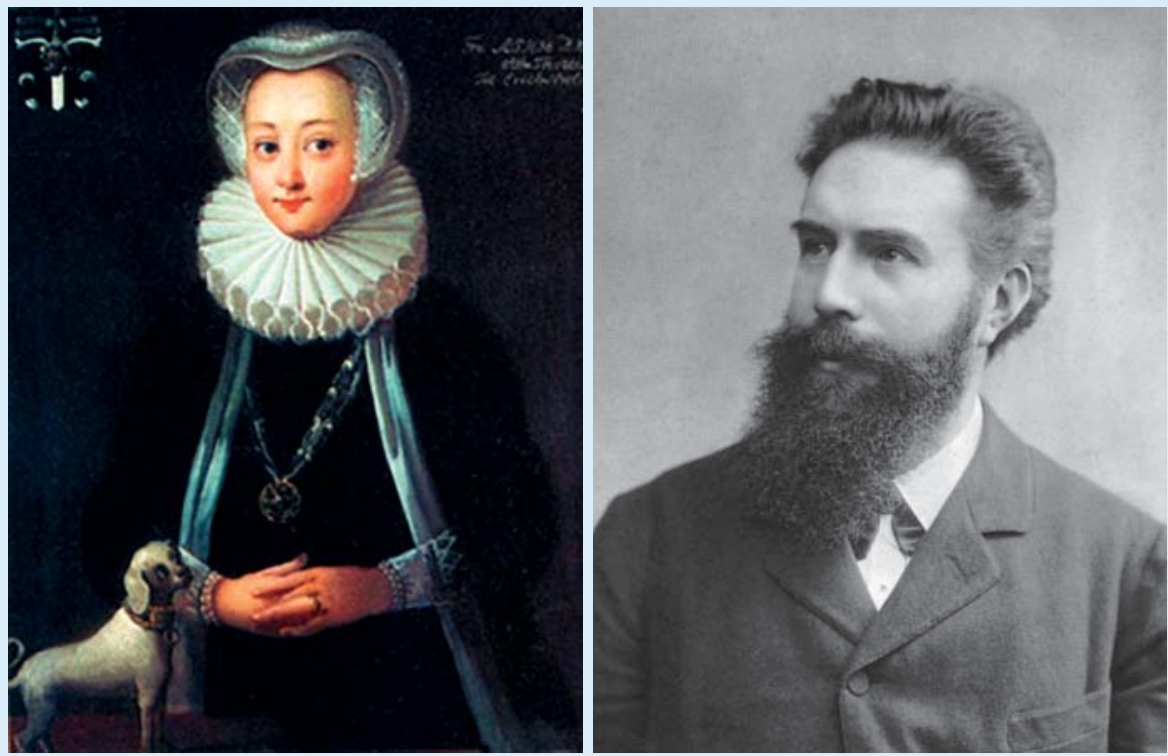

Das Theaterstück wird mit Schülern beider Schulen entwickelt und umgesetzt und am Ende des Schuljahres 2018 aufgeführt. Darüber hinaus ist auch eine Aufführung am Röntgen-Gymnasium in RemscheidLennep geplant.

\section{Infokasten}

Weiterführende Informationen zur Integrierten-Röntgen-Sekundarschule in Berlin-Neukölln finden Sie auf www.roentgen-sekundarschule.de

Auf der Suche nach Gemeinsamkeiten: Sophie Brahe und Wilhelm Conrad Röntgen stehen im Mittelpunkt eines schulübergreifenden Theaterprojekts. 